\title{
Performance of resin-based composite restorations to manage anterior tooth wear
}

The survival and clinical performance of resin-based composite restorations used to treat localised anterior tooth wear C. D. J. Redman, K. W. Hemmings and J. A. Good Br Dent J 2003; 194: 566-572

\section{Objective}

To examine the clinical performance of resin-based composite restorations placed at an increased vertical dimension when used to manage localised anterior tooth wear.

Design

A retrospective analysis of cases treated at a single centre.

\section{Setting}

UK Hospital setting in year 2000.

\section{Subjects and Methods}

Two hundred and twenty five restorations placed in 31 subjects were included. Assessment was made following examination of study casts and projected slides. Modified United States Public Health Services criteria were used and data analysed using the software Statistical Programme for Social Sciences (SPSS). Survival analysis was carried out at two levels, major failure only and all types of failure. Kaplan-Meier survival plots were produced against different variables and modes of failure were also noted.

\section{Results}

Major failure requiring replacement of the restoration was uncommon within the first five years. Minor failure requiring repair or refinishing presented mainly as wear, marginal discolouration or marginal fracture. Median survival was 4 years 9 months when all types of failure were considered. The restorations have good appearance and are well tolerated.

\section{Conclusion}

Placement of resin-based composite restorations at an increased vertical dimension to treat localised anterior tooth wear, has good short to medium term survival. The technique is conservative and relatively easy to maintain.

\section{IN BRIEF}

- Composite resin placed at an increased vertical dimension acts in a similar manner to a 'Dahl' type appliance.

- Composite resin offers a viable treatment option in the management of localised anterior tooth wear.

- There is emerging evidence that composite resin has acceptable clinical performance when used in this way.

- This approach is most conservative and does not preclude other options in the future.

\section{COMMENT}

This relatively limited but important clinical study has attempted to assess the clinical performance of resin-based composite restorations placed in an increased occlusal vertical dimension when used to manage localised anterior toothwear.

The increasing demand to manage toothwear has brought about a number of new restorative clinical techniques over the last decade in particular the use of resin-based composite restorations using the 'Dahl principle' to recreate lost inter-occlusal space. While the process of this clinical technique has been well described in the literature, to date there has been little information available assessing the longer-term performance of these restorations.

The authors have taken the opportunity to review the progress of 225 restorations placed over a period of 5 months to 6 years in 31 patients. The performance of four resin-based composite materials were assessed using well established qualitative criteria and Kaplan-Meier survival plots subsequently produced to compare a number of different variables.

The results seem to indicate that the most common restoration failures were of marginal fracture and discolouration combined with generalised surface wear. Interestingly there were very few catastrophic restoration failures reported. The longer observation periods of up to six years related to the direct resin-based composite materials (Duraphil $n=37$ and Herculite $n=97$ ). Although specific medium survival times were calculated, the results indicated that the directly placed materials perform reasonably well up to the fifth year of follow up whereupon the probability of significant failure increases.

The indirect resin-based composite materials (Herculite $n=18$ and Artglass $n=73$ ) were reported to have performed very well although these restorations had only been in place for a maximum of 3 years. One surprising finding, however, was the significant increased surface wear of the Artglass restorations.

Other variables assessed suggested a higher incidence of restoration failure in patients with a class II division 2 incisal relationship and contrary to what might have been expected there was no significant difference between patients with predominantly erosive wear versus those patients with mainly attrition wear.

Reassuringly there was a high degree of patient satisfaction associated with this technique and orthodontic tooth movements appeared to follow the same pattern and timescale (mean 7 months) as those produced by more conventional 'Dahl' appliances.

In conclusion, the results from this study should continue to encourage the dental profession to use this valuable conservative technique for the restoration of worn anterior teeth.

Paul King, Consultant in Restorative Dentistry, University of Bristol Dental Hospital. 